\title{
Macro Aerodynamic Devices Controlled by Micro Systems
}

\author{
Gwo-Bin Lee*, F.K. Jiang**, Thomas Tsao**, Y.C. Tai ** and C.M. Ho* \\ Mechanical and Aerospace Engineering Department* \\ University of California, Los Angeles \\ LA, CA 90095 \\ 310-825-9993 \\ chihming@seas.ucla.edu \\ Electrical Engineering Department** \\ California Institute of Technology \\ Pasadena, CA 91125 \\ 818-395-8317 \\ yctai@touch.caltech.edu
}

\section{INTRODUCTION}

During the past few years, Micro-ElectroMechanical-Systems (MEMS) has emerged as a major enabling technology across the engineering disciplines. In this study, the possibility of applying MEMS to the aerodynamic field was explored. We have demonstrated that microtransducers can be used to control the motion of a delta wing in a wind tunnel and can even maneuver a scaled aircraft in flight tests. The main advantage of using micro actuators to replace the traditional control surface is the significant reduction of radar cross-sections.

At a high angle of attack, a large portion of the suction loading on a delta wing is contributed by the leading edge separation vortices which originate from thin boundary layers at the leading edge. We used micro actuators with a thickness comparable to that of the boundary layer in order to alter the separation process and thus achieved control of the global motion by minute perturbations.

\section{MEMS BASED TRANSDUCERS}

Separation location can be identified by the profile of the surface shear stress. Measuring surface shear stress in air flow has always been hampered by the lack of sensitive shear stress sensors. We have designed a micro thermal micro shear stress sensor. A polysilicon strip, $2 \mu \mathrm{m} \times 80 \mu \mathrm{m}$, is deposited on top of a thin silicon nitride film and functions as the sensor element. The sensor is placed on a thin diaphragm atop a vacuum chamber. By using sacrificial-layer technique, a vacuum chamber $200 \times 200 \times 2 \mu \mathrm{m}^{3}$ in volume, is placed between the silicon nitride film and silicon substrate. The heat transfer is a function of the normal velocity gradient which is the surface shear stress. In the past, the heating element was directly placed on a substrate. This traditional method resulted in poor sensitivity since air has a very low heat capacity such that most of the heat conducts into the substrate rather than into the air. The vacuum chamber of our new design alleviates the substrate heat transfer. The cavity is formed by sacrificial-layer (PSG, 
phosphosilicate glass) technique. Both the silicon nitride and polysilicon layers are deposited by LPCVD. The aluminum metalization forms the metal leads. The polysilicon resistor is uniformly doped to a low sheet-resistance of typically $50 \Omega / \square$. The measured TCR of the sensor at this doping level is about $0.09 \% /{ }^{\circ} \mathrm{C}$. The typical sensitivity of the sensor with a cavity underneath is $15 \mathrm{mV} / \mathrm{Pa}$, which is about one order of magnitude higher than that without a cavity. Furthermore, sensor arrays on a conformable surface [1] (Fig. 1, Jiang et al. 1997) have been fabricated for distributed sensing on a curved airfoil surface.

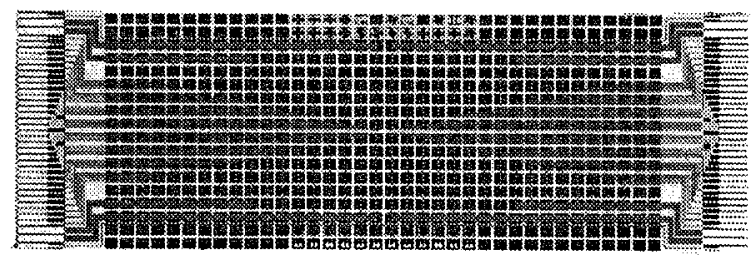

Fig. 1: A $1 \mathrm{~cm}$ x $3 \mathrm{~cm}$ flexible shear stress sensor skin with about 100 sensors on the skin.

In order to withstand the wind load, a high offplane displacement, about $1 \mathrm{~mm}$, is needed to couple the boundary layer flow and high force output, about $1 \mathrm{mN}$, is required for micro actuators. To accomplish this stringent performance, we must first select an actuator with simple configurations so that we at least will not be overburdened with fabrication difficulties.

A flap type actuator was chosen for the design. The flap type actuator is obviously fragile, sensitive to the wind load direction, and bound by many other deficiencies. However, it does serve the purpose of concept demonstration in laboratory tests. Furthermore, the flap actuator has many other applications such as large angle light steering and heat transfer enhancement. The electrostatic force field is unable to achieve these applications. A thin layer of permalloy $(80 / 20 \mathrm{NiFe})$ was deposited on the top of micro actuator to work as the magnet material and electro-magnetic force was applied to drive the actuators. Micromachined permalloy magnetic actuators [2] (Fig. 2, Tsao et al. 1994) were used to manipulate the thin boundary layer near the leading edges. In order to activate these microactuators, a strong permanent magnet with a magnetic flux density of about 1000 Gauss was placed inside the leading edge rod to supply enough magnetic force.

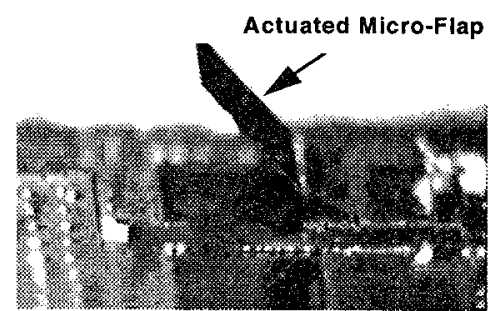

Fig. 2: A magnetically actuated surface micromachined permalloy flap in a B-field of 400 Gauss.

\section{MOMENT OF DELTA WING PRODUCED BY MICRO ACTUATORS}

\section{Experimental Set-up}

An open-type wind tunnel was set up for aerodynamic tests. The test-section was $3 \mathrm{ft} x$ $3 \mathrm{ft}$ and $22 \mathrm{ft}$ long. The maximum speed of this wind tunnel was about $45 \mathrm{~m} / \mathrm{s}$.

A delta wing model with a sweep angle of $56.5^{\circ}$ was employed for this study. The delta wing model had rounded leading edges. Both 
micromachined actuators and mechanical actuators were used. Micromachined actuators and micro shear stress sensors were placed on a cylindrical rod located at the leading edge. The rod could be rotated such that the locations of these transducers could be changed to cover the whole leading edge area. Stripe-type mechanical actuators were installed inside thin slots. The actuators could be extended 1 or $2 \mathrm{~mm}$ out of plane. The location of the actuators could be controlled by rotating the leading edge rod.

A six component force/moment transducer ( AMTI, INC. ) was used to measure aerodynamic forces and moments. Before the testing, this six component force/moment transducer has been fully calibrated.

\section{Rolling Moment Control for Different Angles of Attack}

The delta wing, at different angles of $\operatorname{attack}(\alpha)$, was mounted on a six-component force/moment transducer that records forces and moments in all three axes. Actuators are placed at one of the rounded leading edges. The location of the actuators is indicated by the angle $\theta$ which is zero at the lower surface of the wing (Fig. 3). The torque is measured as a function of $\theta$ for four different angles of attack, $\alpha=20^{\circ}, 25^{\circ}, 30^{\circ}$, and $35^{\circ}$ (Figs. 4.14.4, Figs. 5.1-5.4). The incremental torque generated by the actuator, $M_{r}$, is normalized by $\mathrm{M}_{\mathrm{v}}$, which is the multiplication of the lift force produced by one single vortex and the distance from the center line to the centroid of the half wing. At different operating velocities, the normalized torques more or less collapse to one curve [3] (Lee et al. 1996). For $1 \mathrm{~mm}$ actuators, about $4 \%$ torque is achieved at $\alpha=30^{\circ}$ (Fig. 4-2). The maximum torque increases to more than $15 \%$ for $1 \mathrm{~mm}$ and $40 \%$ for $2 \mathrm{~mm}$ actuation at $\alpha=25^{\circ}$ (Figs. 4-3 \& 5.3). At large $\theta$, the normalized rolling moment becomes negative in most cases. At high $\alpha$, the negative value is fairly small. At $\alpha=25^{\circ}$, the negative torque can be as large as $-30 \%$ for $2 \mathrm{~mm}$ actuation. This negative rolling moment can be employed to obtain an additional rolling moment if actuators are mounted at the proper position at the other leading edge.

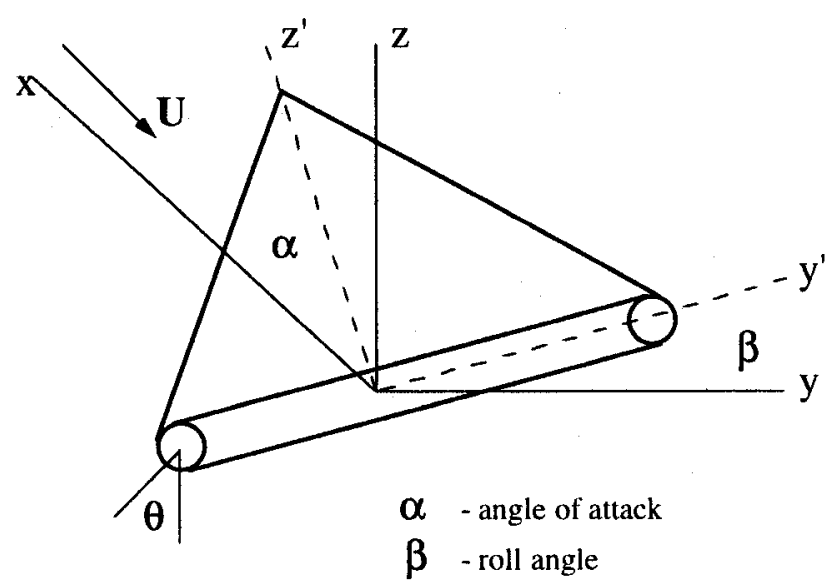

Fig. 3: Coordinate system

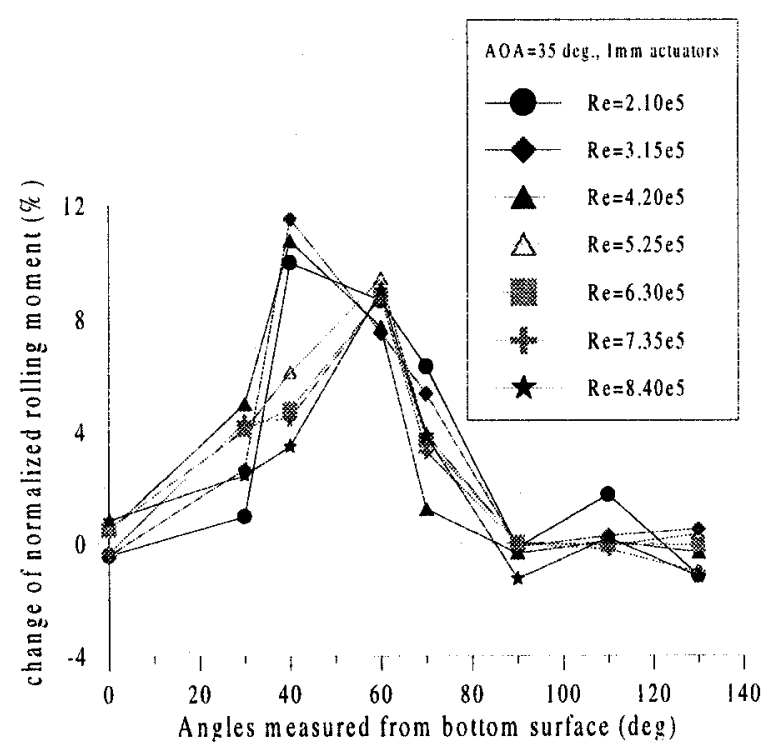

Fig. 4-1: Normalized rolling moment vs. 1 $\mathrm{mm}$ actuation locations at $\mathrm{AOA}=35^{\circ}$ 


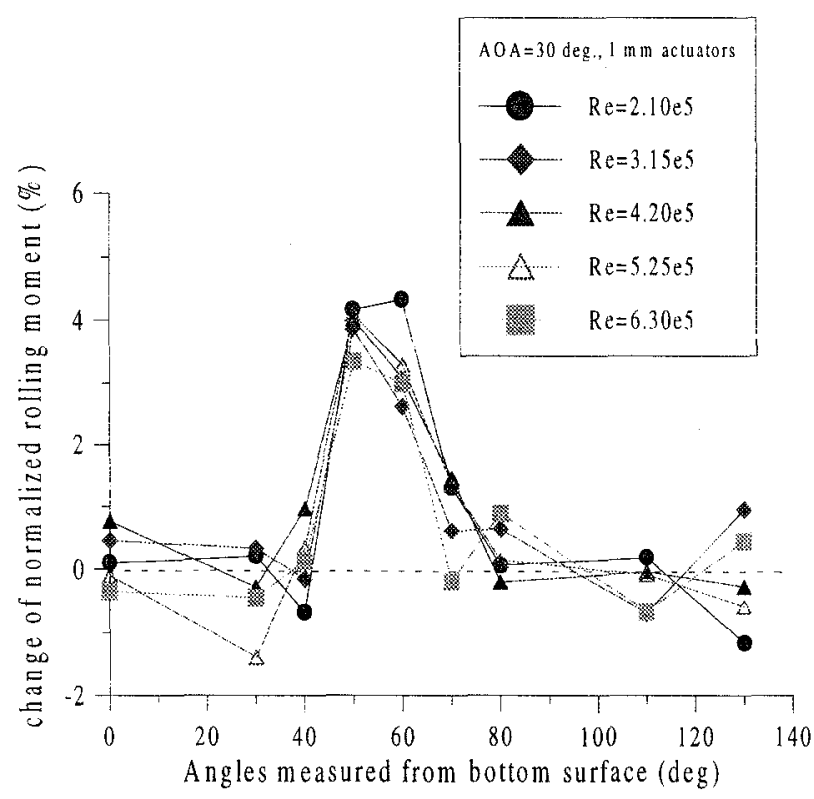

Fig. 4-2: Normalized rolling moment vs. 1 $\mathrm{mm}$ actuation locations at $\mathrm{AOA}=30^{\circ}$

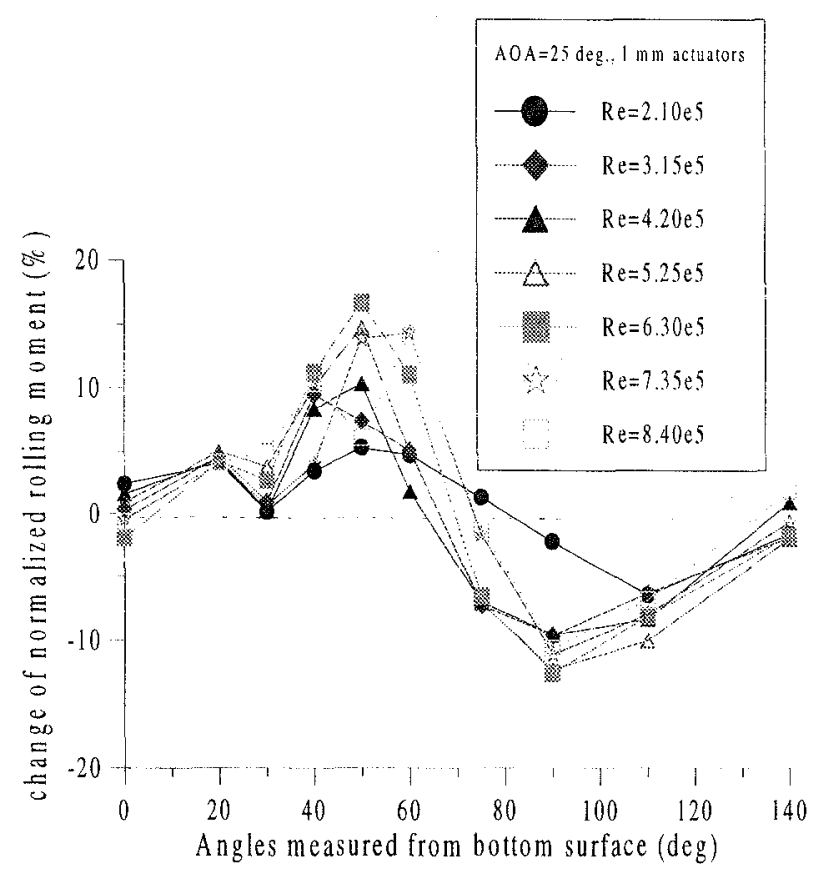

Fig. 4-3: Normalized rolling moment vs. 1 $\mathrm{mm}$ actuation locations at $\mathrm{AOA}=25^{\circ}$

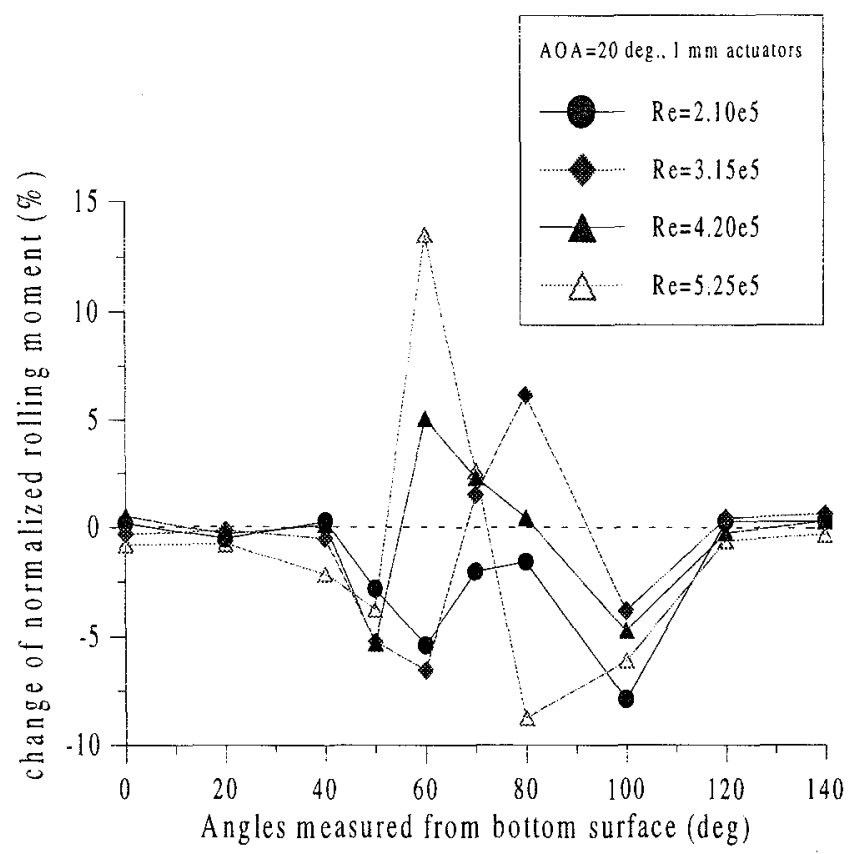

Fig. 4-4: Normalized rolling moment vs. 1 $\mathrm{mm}$ actuation locations at $\mathrm{AOA}=20^{\circ}$

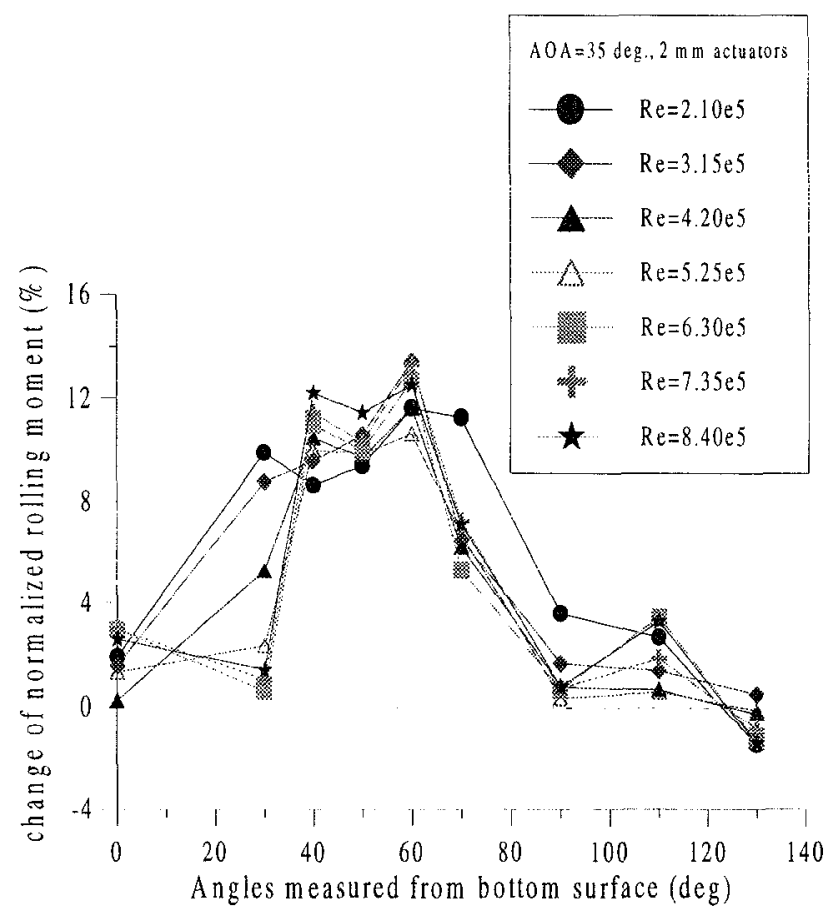

Fig. 5-1: Normalized rolling moment vs. 2 $\mathrm{mm}$ actuation locations at $\mathrm{AOA}=35^{\circ}$ 


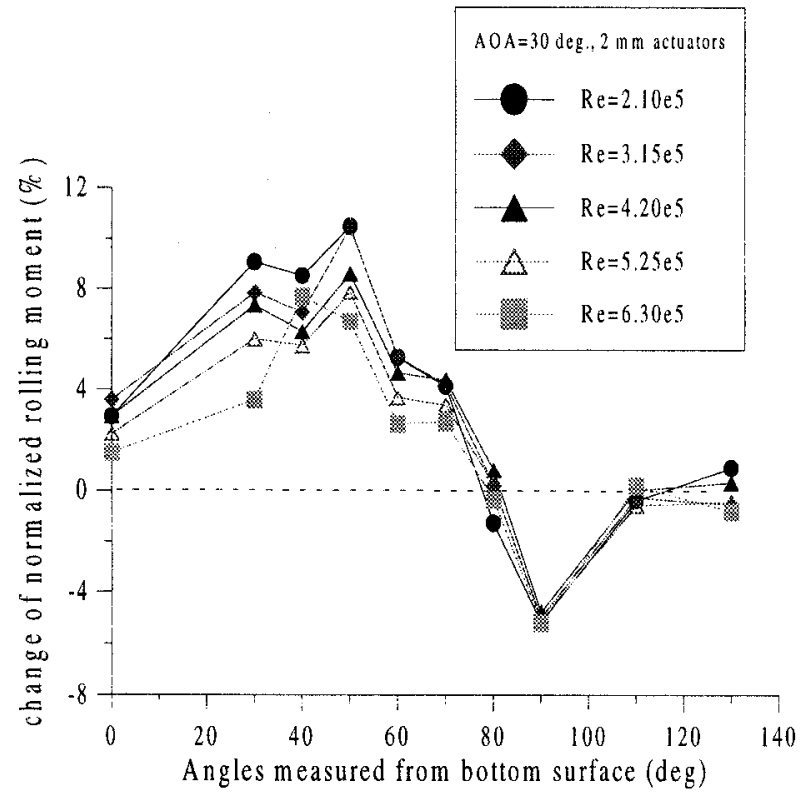

Fig. 5-2: Normalized rolling moment vs. 2 $\mathrm{mm}$ actuation locations at $\mathrm{AOA}=30^{\circ}$

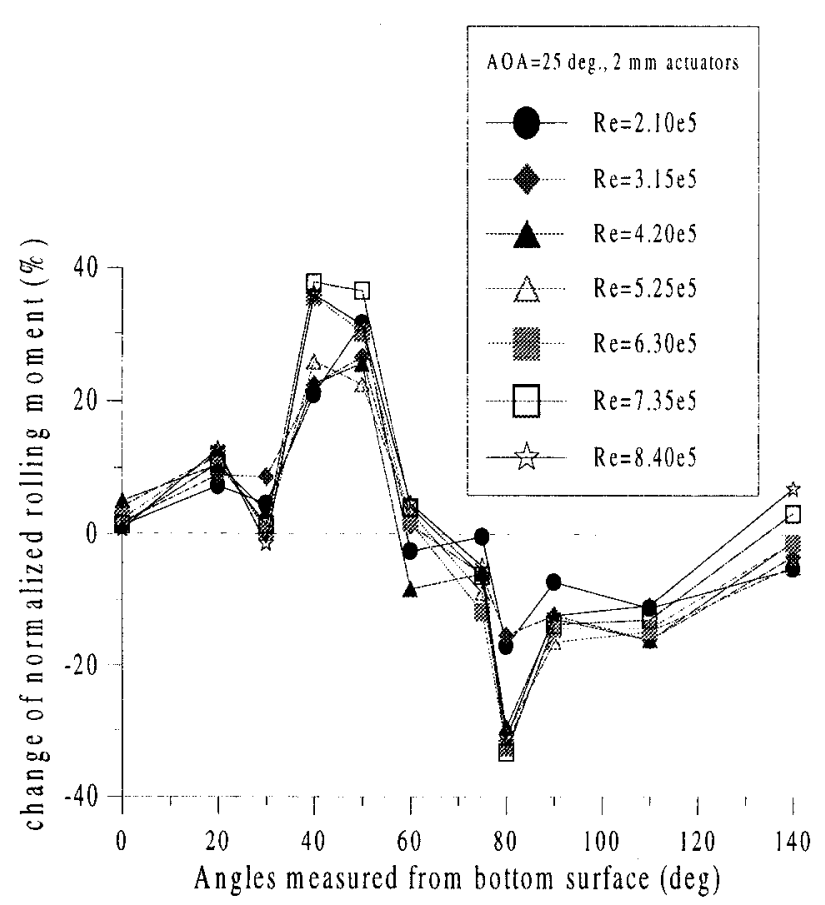

Fig. 5-3: Normalized rolling moment vs. 2 $\mathrm{mm}$ actuation locations at $\mathrm{AOA}=25^{\circ}$

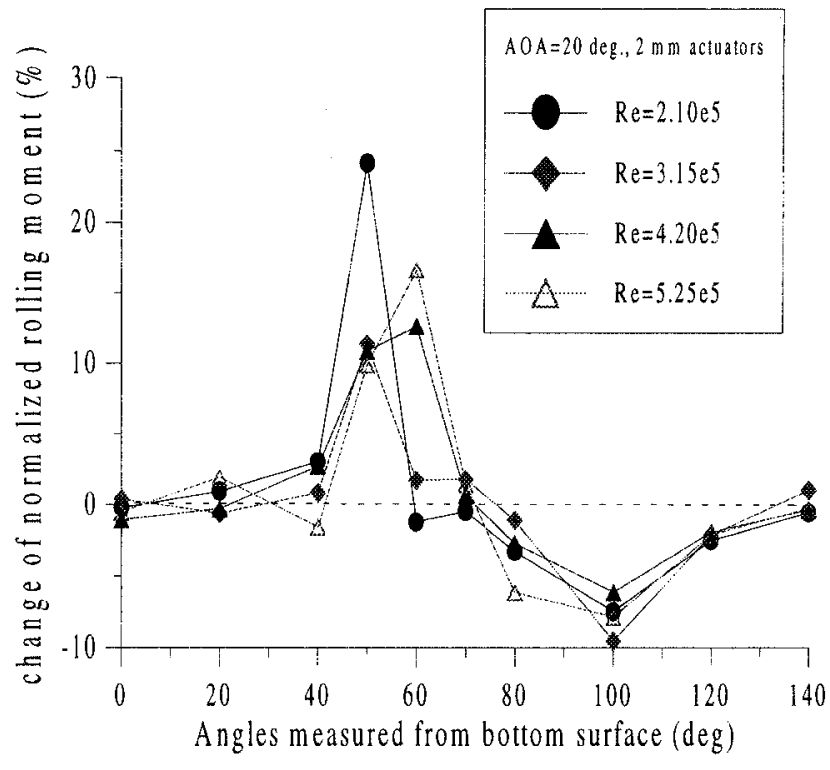

Fig. 5-4: Normalized rolling moment vs. 2 mm actuation locations at $\mathrm{AOA}=20^{\circ}$

\section{Two-Sided Actuation Effect}

The incremental rolling moment reverses its sign when the actuators are placed at large $\theta$. Using this feature, we can get an additional rolling moment if we activate the actuators on both sides of the leading edges at the same time. For example, for a wing at $\alpha=30^{\circ}$ (Fig. 5-2), we mount actuators at the location of $\theta=50^{\circ}$ on one leading edge and also mount actuators at the location of $90^{\circ}$ on the other leading edge. Fig. 6-1 displays the results of the actuation along both leading edges. A $5 \%$ additional rolling moment can be generated by placing actuators at large $\theta$ along the other leading edge. The maximum normalized rolling moment is about $15 \%$ now for $\alpha=30^{\circ}$. Moreover, the summation of individual incremental torques equals the value obtained by the simultaneous actuation along both leading edges. It indicates that modifications of the vortices on both sides of a delta wing by the actuators seem to act independently. 
Much more dramatic results are obtained at smaller angles of attack. For $\alpha=25^{\circ}$, the normalized torque at large $\theta$ can be as much as - 30\%. If the actuators are placed at $\theta=50^{\circ}$ along one leading edge and at $\theta=80^{\circ}$ along the other leading edge, the maximum torque increase reaches a phenomenal level of $70 \%$ (Fig. 6-2). Again, the responses of two vortices to the actuators seem to be independent.

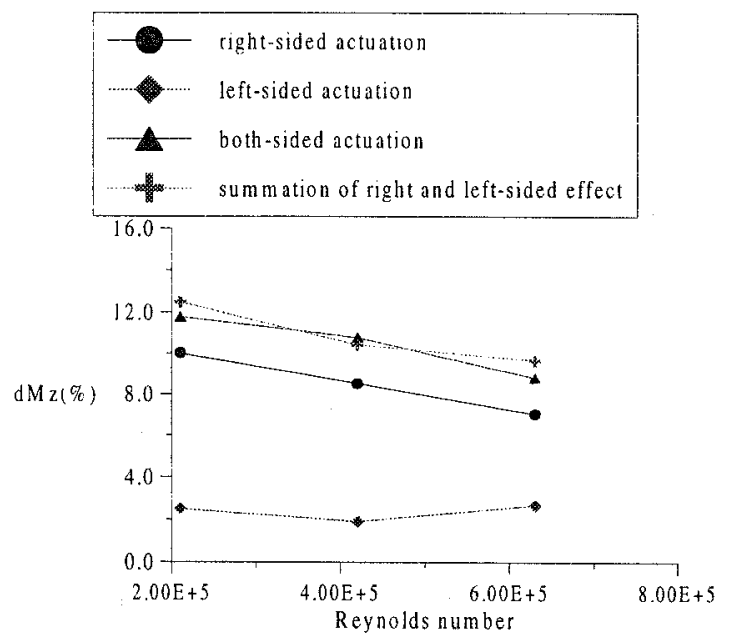

Fig. 6-1: Two-sided actuation effect at $\mathrm{AOA}=30^{\circ}, 2 \mathrm{~mm}$ actuators

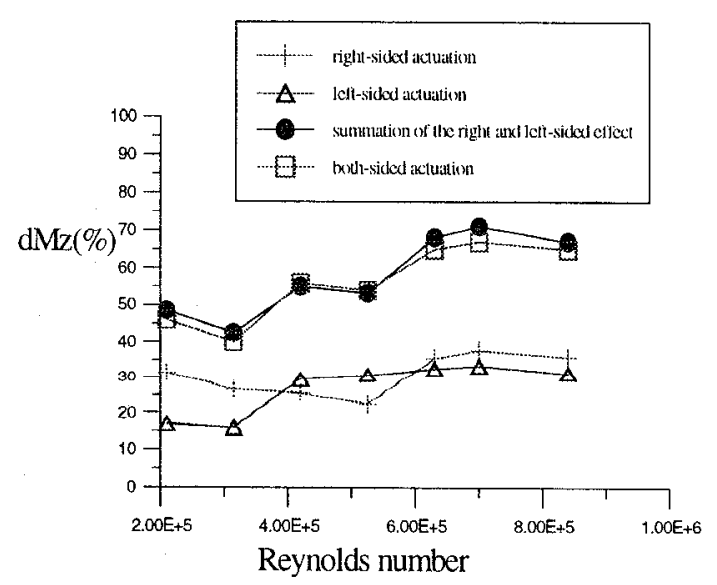

Fig. 6-2: Two-sided actuation effect at $\mathrm{AOA}=25^{\circ}, 2 \mathrm{~mm}$ actuators

\section{Rolling Moment at Non-Zero Roll Angles}

At $\alpha=30^{\circ}$, we examined the rolling moment produced by aerodynamic loading at various roll angles $(\beta)$. This is very important because roll motion is a common mode in aircraft maneuvering. However, our test was in a static state rather than a dynamic rolling motion. The roll angle was varied from $0^{\circ}$ to $58^{\circ}$. The result is shown in Fig. 7. At $\beta=0^{\circ}$, the rolling moment is zero. For roll angles from $0^{\circ}$ to $45^{\circ}$, a positive rolling moment is generated due to aerodynamic loading and a maximum moment occurs at about $\beta=29.5^{\circ}$. For $\beta>45^{\circ}$, the rolling moment becomes negative. For $\beta<45^{\circ}$, the effective sweep angle of one side of the leading edge becomes larger. The position of vortex breakdown is much closer to the trailing edge than the one on the other side so that a positive rolling moment is generated. At a roll angle larger than $45^{\circ}$, the approaching flow accelerates along one side of the pressure surface but decelerates along the other side of the pressure surface. As a result, a negative rolling moment is generated.

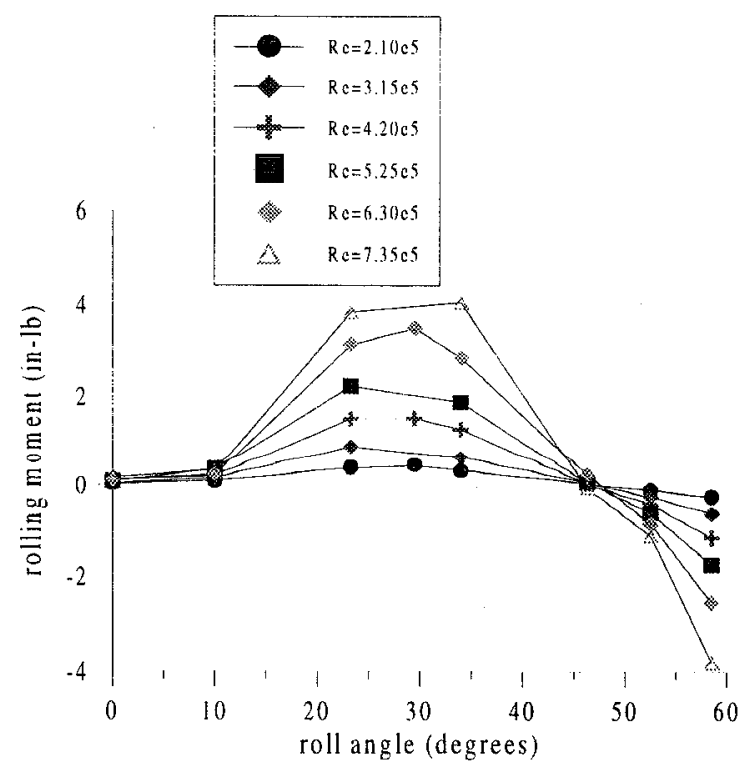

Fig. 7-1: Rolling moment vs. roll angle 


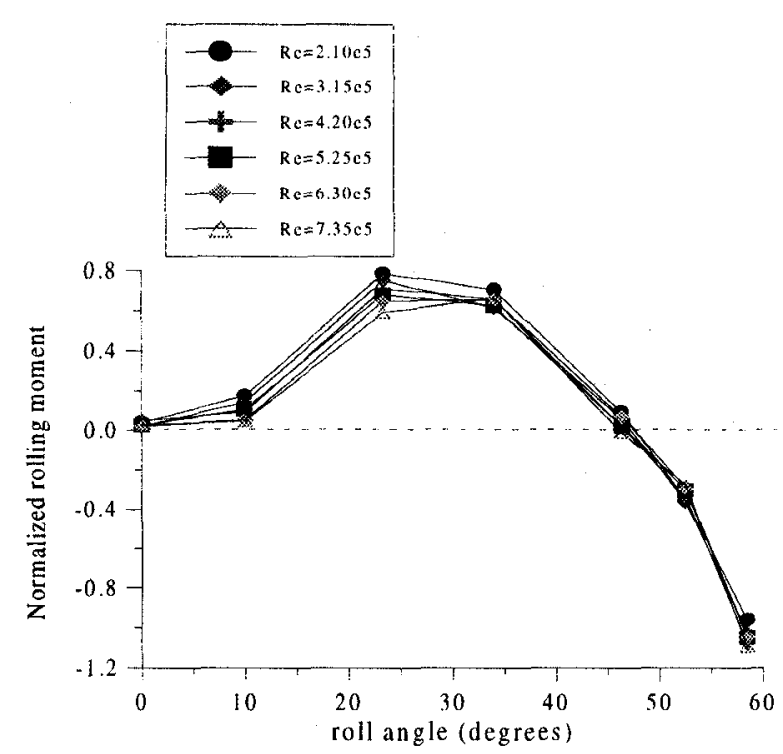

Fig. 7-2: Normalized rolling moment vs. roll angle

Now, we can further appreciate the function of the torque produced at $\beta=0^{\circ}$ by the actuators. It can initiate roll motion and move the wing away from the balanced location. Afterwards, we can take advantage of the large torque generated by the asymmetric flow itself at the non-zero roll angles.

\section{Controllability of Torque at Non-Zero Roll} Angles

The normalized rolling moments collapse for all the measured velocities (Fig. 7-2). It is then interesting to discover the possibility of controlling torque at non-zero roll angles by using miniature actuators. Two roll angles are chosen, $29.5^{\circ}$ and $58.5^{\circ}$, for the reason that the rolling moment due to aerodynamic loading has its maximum and minimum values respectively.

At the roll angle of $29.5^{\circ}$, a large positive rolling moment, about $80 \%$ of the normalized value, is generated. For the first step, we try to reduce the torque by installing micro actuators on both sides of leading edges. Different locations of the actuators were tested to get the optimum results. The results are shown in Fig 8-1. With actuators at the appropriate locations on both sides of the leading edges, the normalized rolling moment can be reduced drastically. Its value can be decreased from 1.0 to 0.05 at a low velocity ( $10 \mathrm{~m} / \mathrm{s}$ ) and from 0.7 to 0.35 at a high velocity $(30 \mathrm{~m} / \mathrm{s})$.

At the roll angle of $58.5^{\circ}$, a large negative rolling moment is generated. Similarly, micro actuators were installed at different locations on both sides of the leading edges to obtain the optimum results. Figure $8-2$ shows that the normalized rolling moment can be increased by the micro actuators. Its value can be changed from -1.0 to -0.4 for velocities ranging from $10 \mathrm{~m} / \mathrm{s}$ to $35 \mathrm{~m} / \mathrm{s}$. In other words, almost $60 \%$ of the negative rolling moment due to aerodynamic loading can be modified by using the micro-actuators.

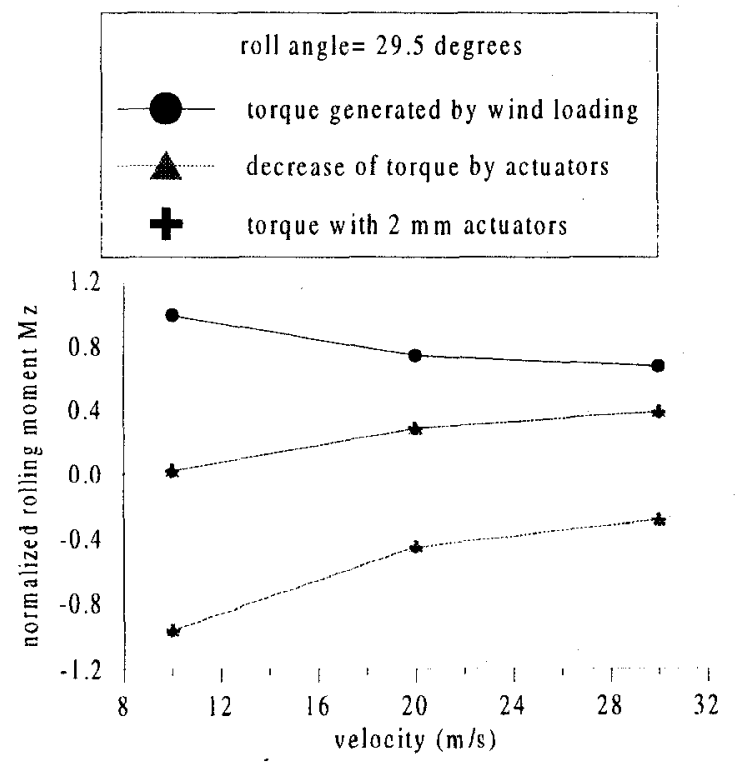

Fig. 8-1: Normalized rolling moment at roll angle of 29.5 degrees with $2 \mathrm{~mm}$ actuators 


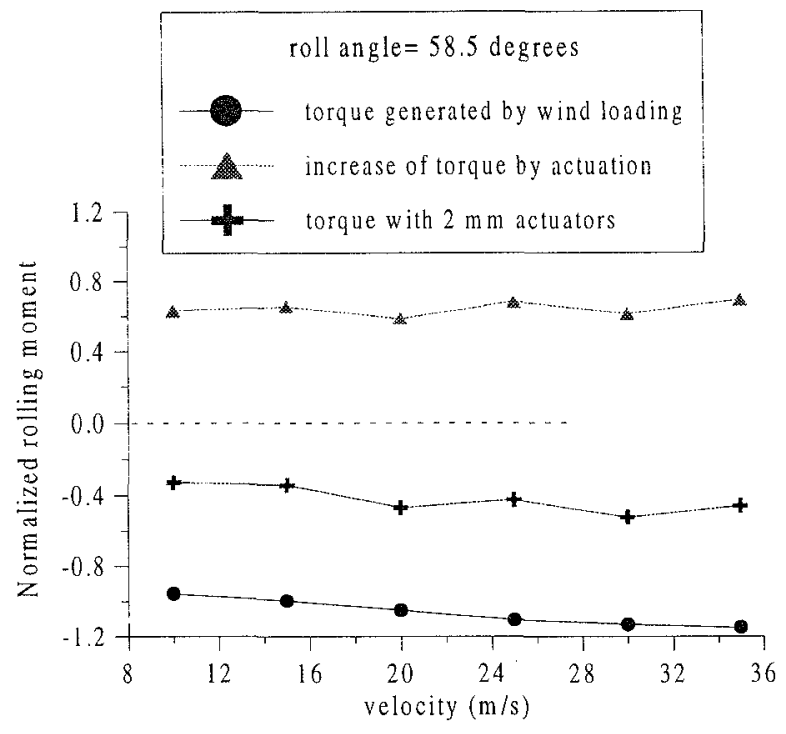

Fig. 8-2: Normalized rolling moment at roll angle of 58.5 degrees with $2 \mathrm{~mm}$ actuators

\section{MANEUVER OF A SCALED MIRAGE III IN FLIGHT TEST}

A $1 / 7^{\text {th }}$ scaled model of a Mirage $\mathrm{III}$ radio controlled aircraft was used to prove the concept. Small stripe-type mechanical actuators were used, rather than micromachined actuators, due to the prior's much higher wind loading in flight tests. The maximum speed of the aircraft was 150 knots and test speeds for the maneuvering cases was about 50 knots. Since the aircraft was not instrumented, difficulties were experienced. However, we did accomplish many exciting flight tests. Many fighter maneuvering modes were achieved. In a high-G barrel roll mode, it was possible to have a $180^{\circ}$ roll made by the aircraft in 0.8 of a second.

\section{ACKNOWLEDGMENT}

This work is support by the DARPA ETO office.

\section{REFERENCES}

[1] Jiang, F., Tai, Y. C., Walsh, K., Lee, G. B., Ho, C. M., "A Flexible MEMS Technology and Its First Application to Shear Stress Sensor Skin", to be published in the proceeding of IEEE MEMS-97 Workshop, Tokyo, Japan, Jan. 26-30, 1997.

[2] Tsao, T., Liu, C., Tai, Y. C. and Ho, C. M., "Micromachined Magnetic Actuator for Active Fluid Control", Application of Microfabrication to Fluid Mechanics, FED-Vol. 197, pp. 31-38,1994, ASME.

[3] Lee, G. B., Ho, C. M., Jiang, F., Liu, C., Tsao, T., and Tai, Y. C., "Distributed Flow Control by MEMS", ASME 1996 International Mechanical Engineering Congress and Exposition, Atlanta, Georgia, USA, Nov. 17-22, 1996.

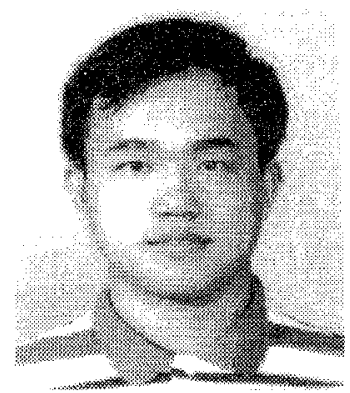

Mr. Gwo-Bin Lee received his $M S$ and $B S$ in Mechanical Engineering from National Taiwan University. He joined Center for Micro Systems at UCLA in 1994. At present, he is a research assistant in Dr. C. M Ho's group and working toward his Ph.D. degree. Mr. Lee's research interests are on the application of micromachined sensors and actuators in flow control.

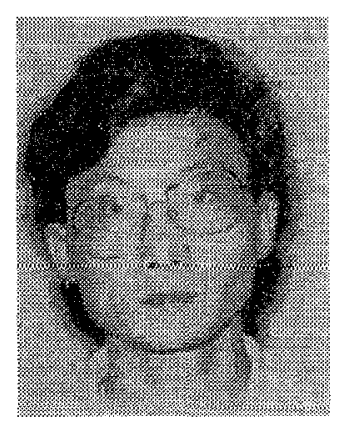

Mr. Fukang Jiang received his $B S$ degree in Physics from Hangzhou University, PRC, in 1984 and MS degree in Electrical Engineering from the California Institute of Technology in 1992, where he is currently working towards his Ph.D. degree on the research and development of MEMS sensors for aerodynamics study. 
$M r$. Thomas Tsao received his $B S$ degree in EE from the University of California at Berkeley in 1992 and MS degree in EE from the California Institute of Technology in 1993. He is currently working toward his Ph.D at Caltech on the research and development of MEMS actuators.

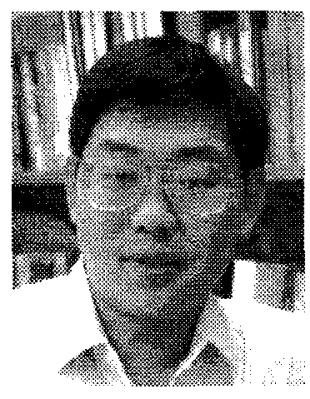

Dr. Yu-Chong Tai is an associate professor at Caltech. He designed and fabricated the first micro motor in the world. He has developed a complete line of transducers for fluid mechanics research. He is the recipient of the prestigious Packard award and NSF young investigator award.

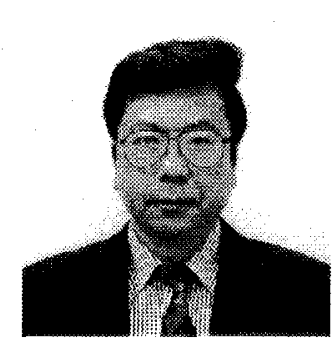

Dr. Chih-Ming Ho is a professor and the Director of Center for Micro Systems at UCLA. His research interests are in MEMS and turbulence. He was elected Fellow of APS and Fellow of AIAA for his contributions in the fundamental understanding of free shear flow under perturbations and pioneering effort of applying MEMS technology to aerodynamic control. 